\title{
Development of a short-form Quadriplegia Index of Function scale
}

\author{
RJ Marino ${ }^{1}$ and JE Goin ${ }^{2}$ \\ ${ }^{I}$ Department of Rehabilitation Medicine, Jefferson Medical College of Thomas Jefferson University, Philadelphia, \\ Pennsylvania and ${ }^{2}$ DataMedix Corporation, Wayne, Pennsylvania, USA
}

Objective: To develop a short-form version of the Quadriplegia Index of Function (QIF) that would be more practical to use than the original version.

Design: Cross-sectional data collected at 6 months post spinal cord injury.

Setting: Regional Spinal Cord Injury Center.

Patients: Consecutive sample of 95 patients with tetraplegia, non-ambulatory at 6 months, admitted to a regional SCI center between December 1987 and August 1992.

Methods: A short-form QIF was developed by using regression analysis to determine the best six items that would predict the sum of the 37 items selected from seven mobility and self-care categories of the original scale. This short-form QIF was evaluated for internal consistency and discriminant validity. Validity of the short-form QIF was assessed by correlation with motor scores and using analysis of variance by motor levels and motor score groupings.

Results: Regression analysis identified the following items as best predictors of the 37-item QIF score: (1) wash/dry hair, (2) turn supine to side in bed, (3) put on lower body clothing, (4) open carton/jar (feeding), (5) transfer from bed to chair, and (6) lock wheelchair. These items explained $99 \%$ of the variance in total scores. Short-form QIF scores (simple sum of the six best items) ranged from 0 to 24 , with a median score of seven, interquartile range $0-16$. Item response patterns were largely dichotomous. Item-total correlations ranged from 0.60 to 0.80; Cronbach's alpha was 0.89 . Spearman correlation coefficient between upper extremity motor score and shortform QIF was 0.82. Analysis of variance indicated that the motor score groupings and motor levels accounted for 63 and 54\% of the variance in short-form QIF scores, respectively. Post hoc analyses indicated that motor levels from C5 to T1 had different mean QIF scores, except for C7 versus $\mathrm{C} 8$. There may be ceiling effects for individuals with low level injuries.

Conclusion: There is significant redundancy in the QIF. Six items, selected from five categories, yield results comparable to the 37-item QIF. The short-form QIF must next be assessed for sensitivity to change. A brief disability measure would improve data quality and completeness, and may permit ongoing collection of observational rather than self-report data.

Keywords: spinal cord injury; tetraplegia; activities of daily living; disability; outcome assessment

\section{Introduction}

Evaluation of disability is important to document improvement in clinical and research settings. Disability status, as measured by the Functional Independence Measure $\left(\mathrm{FIM}^{\mathrm{SM}}\right),{ }^{1}$ is a major component of the Function Related Groups, a classification system being evaluated as the basis for a prospective payment system for rehabilitation. ${ }^{2}$ The third National Acute Spinal Cord Injury Study (NASCIS) ${ }^{3}$ added FIM measures to its design because of questions regarding the clinical significance of motor score changes in the treatment group.

Given the decreasing lengths of stay for patients, it is important to be able to assess disability across settings. For research studies such as the NASCIS

Correspondence: RJ Marino MD, Department of Rehabilitation Medicine, The Mount Sinai School of Medicine, 1425 Madison Avenue, Box 1240, New York, NY 10029, USA trials, it would be preferable to have a capacity measure of disability rather than a performance measure. Performance may lag behind capacity for reasons such as environmental barriers, lack of appropriate equipment, or differing expectations in the home versus the therapy setting. ${ }^{4}$ The FIM is a performance measure, and is collected by observation in the inpatient setting, but by self-report or report of a caregiver or family member after discharge. It would be impractical to observe the entire set of activities that comprise the FIM in a research trial or outpatient clinic. Self-reported measures may underestimate ${ }^{5}$ or overestimate $^{6}$ ability when compared to observed functioning. A valid and reliable brief disability measure would make it practical to observe actual capacity, and would assist in evaluating rehabilitation interventions where performance is inhibited by attenuating factors. 
The Quadriplegia Index of Function (QIF) was developed in 1980 to provide a sensitive functional scale for measuring gains in individuals with tetraplegia during rehabilitation. ${ }^{7}$ The QIF evaluates ten areas of self care and mobility: transfers, grooming, bathing, feeding, dressing, wheelchair mobility, bed activities, bladder program, bowel program, and understanding personal care. The first seven areas are assessed by several items that are scored on a 5point scale. A comparison of FIM and QIF item scoring is found in Table 1. Bowel and bladder programs are scored using criteria specific to the type of program used. Understanding personal care is assessed via multiple choice questions covering ten content areas. The scale has received little attention in the research literature and has not been adopted by rehabilitation centers since its release. This is due in part to the difficulty in administering and scoring the QIF. There is a complex weighting system for calculating category scores from item scores. Importantly, not all items are applicable throughout the period of rehabilitation, particularly when the rehabilitation program extends to the home and outpatient arenas. For example, the feeding item 'prepare simple meals' is not applicable for a hospitalized patient, and the dressing item 'don upper, heavy outdoor clothes' is not pertinent in the summer. These factors, coupled with a lack of scoring instructions in the case of missing items, limit the utility of the scale for measuring changes in function.

There are, however, advantageous features of the QIF compared to other disability measures such as the FIM. An important difference is the 'item scoring' structure of the QIF compared to the 'category scoring' structure of the FIM. The QIF items for selected categories can be seen in Figure 1. The QIF

Table 1 Comparison of QIF and FIM item scoring

\begin{tabular}{lcc}
\hline QIF Score & FIM Score \\
\hline 4 & & 7 \\
3 & Independent & 6 \\
2 & Independent with devices & 5 \\
& Supervision & 4 \\
1 & & 3 \\
& Assistance needed & 2 \\
0 & & 1 \\
\hline
\end{tabular}

EEEDING
Drink from cup/glass
Use spoon/fork
Cut food (meat)
Pour liquids out
Open carton/jar
Apply spreads to bread
Prepare simple meals
Apply adaptive equipment

Apply adaptive equipment*

\section{BED ACTIVITIES}

Supine to prone

Lying to long sitting

Supine to side

Side to side

Maintain long sitting balance
GROOMING

Brush teeth/managing dentures

Brush/comb hair

Shaving (Men)*

Managing tampons or pads (Women)*

BATHING

Wash/dry upper body

Wash/dry lower body

Wash/dry feet

Wash/dry hair

TRANSFERS

Bed to chair

Chair to bed

Chair to toilet/commode

Toilet/commode to chair

Chair to vehicle

Vehicle to chair

Chair to Shower/tub

Tub/shower to chair
DRESSING

Upper indoor clothes on

Upper indoor clothes off

Lower indoor clothes on

Lower indoor clothes off

Socks on/off

Shoes on/off

Fasteners

Upper (heavy) outdoor clothes on*

Upper (heavy) outdoor clothes off*

\section{WHEELCHAIR MOBILITY}

Turn corners

Reverse direction

Lock wheelchair brakes

Move and position in chair

Maintain sitting balance

Propel wheelchair on rough

or uneven surfaces*

Propel wheelchair on an incline*

* items eliminated from analyses

Figure 1 List of items included in selected categories of the Quadriplegia Index of Function 
individually scores feeding tasks such as 'open cartons/ jars', 'apply spreads', 'cut food', and 'pour liquids' that are in the 'Set-Up' level of the FIM. These tasks have a range of difficulty for individuals with tetraplegia, ${ }^{8}$ and the QIF gives appropriate credit for performing them. An individual must perform all of these tasks to score above a ' 5 ' for Eating on the FIM. Therefore the QIF item scoring method allows for finer discrimination in feeding function than the FIM. Compared with the FIM, the QIF feeding score shows a higher correlation to the degree of motor impairment, as measured by the American Spinal Injury Association (ASIA) upper extremity motor score, in individuals with tetraplegia (Spearman rho $=0.90$ versus 0.53$){ }^{9}$

The QIF also has considerable discriminant redundancy. Previous work at the institution of one author suggests that items within self-care and mobility categories of the QIF can be reduced from 45 to 24 without losing discriminating information (unpublished data). This property follows from the multiple measures of the same trait. For example, there is little difference in the functional requirements for 'use a spoon or fork' and 'brush teeth'. This multitrait property provides an opportunity for further item reduction, if an item is considered without regard to its originally defined category.

\section{Methods}

The data used in this project were collected from a single center in a prospective research project to document neurological and functional recovery after spinal cord injury. Subjects were enrolled in this study between December 1987 and August 1992. Trained technicians performed serial manual muscle testing (MMT) on subjects at admission, monthly for 6 months, and at 12 months, using the ASIA standards. ${ }^{10}$ Disability information was collected at $1,2,3$, 6 and 12 months post injury using 45 items from the self care and mobility categories of the QIF, namely transfers, grooming, bathing, feeding, dressing, wheelchair mobility, bed activities (Figure 1). The QIF data was collected by in-person interview of subjects. The study excluded patients with concomitant upper extremity fractures, head injuries, or psychiatric disturbances that interfered with MMT or QIF testing.

Subjects included 95 of 99 patients from the database with tetraplegia on initial evaluation, who were non-ambulatory at 6 months post injury. Subjects with limited ambulatory ability (exercise or household only) were included. Ambulatory subjects were not included because the QIF assesses only wheelchair mobility, not walking. Data from four patients were eliminated because of incomplete data.

The present investigation utilized 6-month neurological and disability information from the database. The 6-month time period was chosen because by this time most subjects had completed rehabilitation and were no longer wearing orthopedic appliances (eg
Halo orthoses, cervical collars). Using initial and 6month neurological data, motor levels and Frankel grades were assigned to each subject in accord with the 1992 ASIA standards. ${ }^{11}$ The motor level is defined as the most caudal segment of the spinal cord with normal motor function, and is determined separately for the right and left sides of the body. An upper extremity motor score (UEMS) was calculated for each subject by adding the scores of the ten key upper extremity muscles.

Data cleaning and item reduction of the QIF involved several steps. As noted above, only a subset of the original QIF categories were used in the database. The categories 'bowel program' and 'bladder program' were not used because the criteria would not be consistent across time periods. The category 'understanding personal care' was not collected since it was not related to motor functioning, and would not discriminate among levels of injury. This left 45 items in seven categories for further analysis. Next, item endorsement was assessed. Eight items with a large proportion of missing responses were identified, including two genderspecific items from the grooming category (shaving, managing tampons). These eight items were dropped, leaving 37 items in the database (Figure 1). For these items, values for missing or non-applicable responses were imputed. Imputed values were determined by decision rules established prior to data analysis. These rules attempted to match the structure of the QIF. For example, the score for 'use spoon/fork' was used if 'brush teeth' was missing. If no acceptable substitute was available, the average of the other items in that category was used. Imputed values represented only $2.7 \%$ of the QIF data.

Thirty-seven items were reduced to six items using regression analysis. Prior work had suggested that the six feeding items might be able to discriminate among the various levels of tetraplegia. ${ }^{9}$ However, it was felt that the items in the feeding category might not contain a sufficient range of difficulty for all levels of tetraplegia. Regression analysis was employed as a tool for item reduction, to identify the best six items without regard to original category. Six items were chosen to correspond to the number of items retained in the feeding category, and represented a ratio of the number of subjects to the number of predictor variables of approximately $15: 1(90 / 6)$, which is adequate for prediction purposes.

The regression approach chosen for exploratory model building was to identify the six items which provided best prediction of the 37-item QIF scores, where best prediction was interpreted as providing the highest squared correlation between the predicted $37-$ item QIF scores and the observed 37-item QIF scores. The 37-item QIF score was taken as the dependent variable in the model, and represents a summatedrating score while the independent variables were the 37 individual items. The summated-rating score was assumed to represent a continuous variable on an 
interval scale, and each of the individual independent predictors were considered given values of an underlying continuous interval scale. The exploratory regression model, with these assumptions, was implemented using the regression procedure in SAS (Cary, NC, USA) with the best option for variable selection. The regression model was constrained by omitting certain items from the analysis. Because the transfer items were highly correlated, only 'transfer bed to chair' was included in the model. Transfers to/ from vehicle were not included because of anticipated difficulty observing this activity in most settings, and transfers to/from toilet because this activity is sometimes not applicable due to architectural barriers. One other item, 'maintaining sitting balance', was excluded because of anticipated difficulty objectively scoring this activity.

A separate regression analysis was performed to predict 37-item QIF score (sum of 37-item-scores) using either the best six items or the feeding items. An examination of residuals was performed to determine how closely predicted scores matched actual values. A short-form QIF was obtained by summing the scores of the six best items. For the sake of simplicity, it was decided not to weight the items, as had been done in the original QIF.

\section{Psychometric assessment of short-form scale}

Distribution of item responses, and means and standard deviations (SD) of the best six items were examined. Ideally, item responses should be approximately normally distributed. Large deviations from a normal distribution call into question the meaning of average scale scores, and suggest that the response structure should be examined. Outlier items, defined as items whose mean value was two SD above or below the average item mean, were identified. Items with outlier SD were similarly identified. Items with low or high means and small SD may indicate a ceiling or floor effect.

Internal consistency was examined using Cronbach's alpha $^{12}$ and item-total correlations. The value for Cronbach's alpha should be above $0.70^{13}$ However, values above 0.90 suggest a high level of redundancy in the scale. ${ }^{14}$ An item-total correlation, which is the correlation of an item score with the sum of the remaining items, should be above $0.20 .^{14}$
To test the discriminant validity of the scale, the 6month MMT data was used. The correlation of the UEMS with the short-form QIF was determined. Mean short-form QIF scores were calculated for each motor level, using the best motor level (BML) in each subject. ${ }^{15}$ For this analysis, the $\mathrm{C} 4$ and $\mathrm{C} 5$ levels were combined and unilateral motor levels greater than $\mathrm{T} 1$ were grouped with the T1 data. Subjects were also arranged into five groups based on UEMS scores $(0-$ 10, 11-20, etc.), and mean QIF scores were calculated for each group. Analysis of variance was used to compare the amount of variability of short-form QIF scores explained by the motor groupings. Post-hoc comparisons of differences in mean QIF scores among motor levels or motor score groups were made using Fisher's least-significant-difference test. Because motor incomplete subjects may have less variability in disability across levels, ${ }^{16}$ the validity analyses were repeated using only the 76 motor complete subjects. To check the decision not to weight the individual items for the short-form QIF, the analysis of variance was repeated using the motor level and motor score groupings and a weighted QIF. The weightings were those derived from the regression analysis (Table 2). All analyses were performed using SAS version 6.08 (SAS Institute, Cary, NC, USA).

\section{Results}

The subjects included 85 men and 10 women, ranging in age from $16-68$ years $($ mean $=31.2, \quad \mathrm{SD}=13.2)$. Initially there were 60 subjects classified as Frankel A, $23 \mathrm{~B}, 11 \mathrm{C}$ and $1 \mathrm{D}$. At 6 months there were 57 Frankel A subjects, 19 B, $7 \mathrm{C}$ and $12 \mathrm{D}$. None of the Frankel D subjects were community ambulators. The 6-month motor score in the upper extremities ranged from $2-50$, with a mean of $23.4(\mathrm{SD}=13.4)$, while the 37-item QIF scores spanned the entire range from $0-$ 148 , with a mean of $55.6(\mathrm{SD}=50.0)$ and a median score of 42. Although all subjects initially were classified as tetraplegic, four recovered normal strength in all upper extremity key muscles by 6 months. Three of these subjects had 6-month sensory levels below $\mathrm{T} 1$, and would be classified as paraplegic at this time.

Regression analysis of individual items to predict 37-item QIF score (sum of 37 item scores) was performed using a subset of QIF items, as described

Table 2 QIF score (37-item) regression analysis, $\mathrm{R}^{2}=0.99$

\begin{tabular}{lcccrc}
\hline Effect & Coefficient & Std Error & Tolerance & $\mathrm{t}$ & $\mathrm{P}$ \\
\hline Wash/dry hair & 4.286 & 0.605 & 0.308 & 7.082 & $<0.001$ \\
Turn supine to side in bed & 5.696 & 0.635 & 0.215 & 8.974 & $<0.001$ \\
Put on lower body clothing & 6.556 & 0.790 & 0.324 & 8.298 & $<0.001$ \\
Open carton/jar & 4.404 & 0.609 & 0.334 & 7.229 & $<0.001$ \\
Transfer from bed to chair & 8.813 & 0.875 & 0.220 & 10.075 & $<0.001$ \\
Lock wheelchair & 6.887 & 0.441 & 0.303 & 15.618 & $<0.001$ \\
\hline
\end{tabular}


in the Methods. The regression analysis identified the following six items as best:

(1) wash/dry hair,

(2) turn supine to side in bed,

(3) put on lower body clothing,

(4) open carton/jar (feeding),

(5) transfer from bed to chair, and

(6) lock wheelchair.

Regression analysis indicated that these items explained $99 \%$ of the variance in total scores (Table 2). The QIF scores using the regression coefficients (Table 2) were within ten points of actual values in 80 of 95 subjects (84\%), and within 15 points in 90 of $95(95 \%)$. In comparison, regression analysis using the feeding items explained $96 \%$ of the variance in 37-item QIF scores. However on an individual basis errors were larger using the feeding items. Only 59 of 95 subjects $(62 \%)$ had predicted scores within ten points of actual values,

Table 3 Correlation of short-form QIF item scores with 37item QIF score

\begin{tabular}{lcc}
\hline Item & Pearson & Spearman \\
\hline Wash/dry hair & 0.784 & 0.758 \\
Turn supine to side in bed & 0.825 & 0.844 \\
Put on lower body clothing & 0.794 & 0.700 \\
Open carton/jar & 0.772 & 0.730 \\
Transfer from bed to chair & 0.879 & 0.845 \\
Lock wheelchair & 0.722 & 0.830 \\
Short-form QIF & 0.987 & 0.978 \\
\hline
\end{tabular}

and only 75 had scores within 15 points. The Spearman correlation coefficient between the shortform QIF score and 37-item QIF score was 0.978. Each of the six items was positively correlated with the 37-item QIF score (Table 3).

Item score distributions, medians, means and SD are given in Table 4. Score distributions were generally bimodal, with the most frequent score being either 0 or 4 . Item means ranged from 0.79 2.64 , and SD ranged from $1.53-1.86$. There were no outlier items.

Scores for the short-form QIF ranged from 0-24, with a mean of 9.0 and SD of 8.3 The median score was seven, with an interquartile range from $0-16$. Item-total correlations for the short-form QIF ranged from $0.60-0.80$. The value for Cronbach's alpha was 0.89 .

Spearman correlation of the UEMS with the shortform QIF was 0.824. Discriminant validity of the short-form QIF was generally supported by the progression of scores by motor level (Table 5) and motor score (Table 6). The mean score increased with each motor level, except for $\mathrm{C} 7$ and $\mathrm{C} 8$, which were similar. For the motor score groups, mean QIF scores were significantly different for all pairs of groups except groups ' $21-30$ ' compared to ' $31-40$ '. Analysis of variance indicated that the BML grouping explained $54 \%$ of the variance in short-form QIF scores. The UEMS grouping explained $63 \%$ of the variance in short-form QIF scores. Use of a weighted QIF score did not improve the amount of variance explained.

When the analysis was limited to the 76 motor complete subjects, the BML and UEMS groupings explained 61 and $66 \%$ of the variance in QIF scores,

Table 4 Short-form QIF item distributions and summary statistics

\begin{tabular}{|c|c|c|c|c|c|c|c|c|c|}
\hline \multirow[b]{2}{*}{ Item } & \multicolumn{5}{|c|}{ QIF Item Score } & \multirow[b]{2}{*}{ Mode } & \multirow[b]{2}{*}{ Median } & \multirow[b]{2}{*}{ Mean } & \multirow[b]{2}{*}{$S D$} \\
\hline & 0 & 1 & 2 & 3 & 4 & & & & \\
\hline Lock Wheelchair & 28 & - & 2 & 13 & 52 & 4 & 4 & 2.64 & 1.77 \\
\hline Turn supine to side in bed & 43 & 7 & 1 & 9 & 35 & 0 & 1 & 1.85 & 1.86 \\
\hline Wash/dry hair & 61 & - & 3 & 1 & 30 & 0 & 0 & 1.36 & 1.86 \\
\hline Open containers & 64 & 1 & 1 & 2 & 27 & 0 & 0 & 1.23 & 1.82 \\
\hline Transfer bed to chair & 55 & 12 & 8 & 4 & 16 & 0 & 0 & 1.09 & 1.54 \\
\hline Put on lower indoor clothing & 71 & 7 & - & - & 17 & 0 & 0 & 0.79 & 1.53 \\
\hline
\end{tabular}

Table 5 Mean short-form QIF scores by best motor level group

\begin{tabular}{lccrr}
\hline Best motor level & \multicolumn{2}{c}{ Total Group $(\mathrm{n}=95)$} & \multicolumn{2}{c}{ Frankel $A$ or B $(\mathrm{n}=76)$} \\
Number & Mean $(S D) *$ \\
C4/5 & 33 & $2.5(4.4)$ & 30 & $2.2(3.9)$ \\
C6 & 25 & $7.4(6.5)$ & 20 & $6.5(6.0)$ \\
C7 & 19 & $13.6(6.7)$ & 11 & $11.5(6.1)$ \\
C8 & 7 & $13.1(7.0)$ & 6 & $14.7(6.3)$ \\
T1 & 11 & $21.0(4.9)$ & 9 & $21.0(5.4)$ \\
\hline
\end{tabular}

*All pairwise values differ except for C7:C8, by Fisher's least-significant-difference test 
respectively (data not shown). While there was a greater separation of mean QIF scores between subjects whose BML was $\mathrm{C} 7$ versus $\mathrm{C} 8$, this difference did not reach statistical significance. There was little variability in scores for the $\mathrm{T} 1+\mathrm{BML}$ group; $75 \%$ of the scores were between 22 and 24 .

Profiles of ability to perform QIF items without assistance (item score 3 or 4 ) by BML are presented in Table 7. These results indicate that there is a hierarchical structure to the short-form QIF. The number of items completed by at least half the subjects increases with successive BML, ranging from no items for C5 individuals to all six items for T1 individuals. For each item, the per cent successful increases with each level except for the C8 group, which may have insufficient numbers for an accurate assessment. Examining the distribution of short-form QIF scores by BML, three outlier subjects were found. The first was an 18 year old male with a Frankel B, C5 motor level bilaterally and an UEMS of 17, who was unusually functional for his level (short-form QIF 17 out of 24). His motor score is more typical of a C6 injury, which may explain his high level of functioning. The second outlier patient was a 29 year old female with an asymmetric injury, motor level T1 on the right, C6 on the left, and UEMS of 43, who was hopsitalized at the time of the 6 month assessment. Her lower than expected QIF score (7 out of 24) may have been due to the hospitalization. Unfortunately, she did not return for a 12 month assessment. The third outlier was a 56 year old gentleman, Frankel D, with a motor level of C5 on the left, C4 on the right, and an UEMS of 33.5. His short-form QIF score of 16 is high for his motor level, but not unusual for his UEMS.

\section{Discussion}

The results of this study demonstrate that a short-form QIF, consisting of six items, can be constructed that provides discriminatory disability information in nonambulatory individuals with tetraplegia. Six items explained $99 \%$ of the variance of the 37-item QIF score. The short-form OIF retains good internal consistency characteristics with less redundancy.

Item score distributions were non-normal, suggesting that summed scores may not be ideal. A dichotomous response structure may be indicated. However the exact cut-point is unclear. For certain items the 'independent with devices' response was endorsed relatively often, while for others the 'assistance needed' option was selected (Table 4). In addition, responses that are less frequently selected at 6 months may be more frequently endorsed at different time periods. Therefore it is premature to recommend a reduced response structure for QIF items.

The original QIF used weightings of individual items to determine a total score. No rationale for the weightings was given in the original article describing the QIF. ${ }^{7}$ In this study, use of item weights did not add to the ability of the short-form QIF to discriminate among individuals based on motor level or motor score. These findings support the decision not to weight items, thereby simplifying use of the short-form QIF.

The short-form QIF was unable to differentiate between individuals with $\mathrm{C} 7$ and $\mathrm{C} 8$ tetraplegia. This may be due to the small number of subjects in the C8 group, or a lack of appropriate discriminating items. There were only seven subjects in the C8 BML

Table 6 Mean short-form QIF scores by motor score group

\begin{tabular}{lccrr}
\hline UEMS score & \multicolumn{2}{c}{ Total group $(\mathrm{n}-95)$} & \multicolumn{2}{c}{ Frankel $A$ or $B(\mathrm{n}=76)$} \\
Number & Mean $(S D) *$ & $1.0(2.2)$ \\
$0-10$ & Number & $1.0(2.2)$ & 21 & $4.6(4.5)$ \\
$11-20$ & 21 & $4.4(4.5)$ & 23 & $11.4(6.3)$ \\
$21-30$ & 26 & $11.8(6.4)$ & 15 & $13.5(5.6)$ \\
$31-40$ & 21 & $14.7(6.8)$ & 4 & 13 \\
$41-50$ & 12 & $19.6(5.9)$ & $13.2(6.2)$ \\
\hline
\end{tabular}

$\mathrm{QIF}=$ Quadriplegia Index of Function; UEMS = upper extremity motor score. *All pairwise values differ except for $21-30: 31-$ 40

Table 7 Percentage of subjects able to complete item independently (QIF score 3 or 4)

\begin{tabular}{|c|c|c|c|c|c|c|c|}
\hline \multirow[b]{2}{*}{$\begin{array}{l}\text { Best motor } \\
\text { level }\end{array}$} & \multirow[b]{2}{*}{$\begin{array}{c}\text { Number of } \\
\text { subjects }\end{array}$} & \multirow[b]{2}{*}{$\begin{array}{c}\text { Lock } \\
\text { wheelchair }\end{array}$} & \multicolumn{2}{|r|}{ QIF Items } & \multirow[b]{2}{*}{$\begin{array}{c}\text { Open } \\
\text { containers }\end{array}$} & \multirow[b]{2}{*}{$\begin{array}{l}\text { Transfer bed } \\
\text { to chair }\end{array}$} & \multirow[b]{2}{*}{$\begin{array}{l}\text { Put on lower } \\
\text { indoor clothing }\end{array}$} \\
\hline & & & $\begin{array}{l}\text { Turn supine to } \\
\text { side in bed }\end{array}$ & $\begin{array}{c}\text { Wash/dry } \\
\text { hair }\end{array}$ & & & \\
\hline $\mathrm{C} 4 / 5$ & 33 & 36 & 9 & 12 & 6 & 0 & 3 \\
\hline C6 & 25 & 76 & 36 & 24 & 24 & 4 & 8 \\
\hline C7 & 19 & 89 & 84 & 53 & 42 & 37 & 21 \\
\hline $\mathrm{C} 8$ & 7 & 86 & 71 & 57 & 57 & 29 & 14 \\
\hline $\mathrm{T} 1+$ & 11 & 100 & 100 & 73 & 82 & 91 & 82 \\
\hline
\end{tabular}


group. The profile of scores by level (Table 7) indicates that for several items performance of the C8 group lagged that of the C7 group. Zafonte et al ${ }^{8}$ found that all five subjects with a BML of C8 could open containers independently, compared to two out of seven in this study. This suggests that the C8 individuals in this study may not have been typical of individuals with C8 BML. Data from the model SCI systems suggests that the FIM can differentiate between these two levels. Mean discharge FIM scores in the National SCI database were 53.2 for individuals with $\mathrm{C} 7$ injuries and 65.1 for those with C8 level lesions. ${ }^{16}$

The high mean score for subjects whose BML is T1 suggests that there may be a ceiling effect for individuals with low level tetraplegia, particularly those with minimal deficits who would be expected to fully recover strength in the hand muscles. An item of greater difficulty may be necessary for the shortform QIF to detect improvement in this group.

Previous work using the feeding category of the QIF demonstrated increased sensitivity over the FIM in discriminating individuals with various levels of tetraplegia.' The 'set up' items in the feeding category-cut food, open carton, etc-have been shown to distinguish between some levels of tetraplegia. ${ }^{8}$ It was hypothesized that the feeding items alone might be able to replace the entire QIF. However, the results of this study indicate that the feeding items do not contain an adequate distribution of difficulty to substitute for the QIF. While the total amount of variance explained was similar using the feeding items or the best six items, on an individual basis large deviations of predicted scores from true scores were more common using the feeding items. Rather, selecting items from several different categories improves the accuracy of disability estimation.

Further work must be done before the reduceditem QIF can be recommended for assesssment of disability. This study looked at one time period, namely 6 months post injury, to reduce the QIF items. Assessment measures can be used for either discriminative or evaluative functions. A discriminative instrument seeks to distinguish between individuals at a given point in time; an evaluative instrument seeks to identify change within individuals over time. ${ }^{17}$ Items may be better suited for one or the other purpose. For example, the ability to walk can discriminate between those with AIS grade $\mathrm{A}$ and AIS grade D SCI. However, walking ability would not be a good evaluation item for those with AIS grade A injuries, because ability to walk would not be expected to change within this group.

\section{Conclusion}

There is considerable redundancy in the QIF. Six items, selected from five categories, yield results comparable to a 37-item scale. Further work is needed to determine if the same items evaluate change effectively, and if addition of a few more items would improve discriminative ability. Using a limited number of items to assess disability should improve data quality and completeness, and may permit ongoing collection of observational rather than self-report data.

\section{Note added in proof}

A preliminary version of this material was presented at the 22nd annual meeting of the American Spinal Injury Association, Seattle, WA, April 22-24, 1996

\section{Acknowledgements}

This work was supported in part by K11 grant \#HD01020 from the National Center for Medical Rehabilitation Research, National Institutes of Health.

\section{References}

1 Guide for the Uniform Data Set for Medical Rehabilitation (Adult FIM), Version 4.0, Buffalo, NY 14214: State University of New York at Buffalo; 1993.

2 Stineman MG et al. A Prototype Classification System for Medical Rehabilitation. Washington DC: American Rehabilitation Association, 1994.

3 Bracken MB et al. Administration of methylprednisolone for 24 or 48 hours or tirilizad mesylate for 48 hours in the treatment of acute spinal cord injury: results of the third national acute spinal cord injury randomized controlled trial. JAMA 1997; 277: 1597 1604.

4 Jette AM. Physical disablement concepts for physical therapy research and practice. Phys Ther 1994; 74: 380-386.

5 Harris BA, Jette AM, Campion EW, Cleary PD. Validity of selfreport measures of functional disability. Topics in Geriatric Rehabilitation 1986; 1: $31-41$.

6 Skruppy M. Activities of daily living evaluations: is there a difference in what the patient reports and what is observed? Physical and Occupational Therapy in Geriatrics 1993; 11(3): 13 25

7 Gresham GE et al. The quadriplegia index of function (QIF): Sensitivity and reliability demonstrated in a study of thirdy quadriplegic patients. Paraplegia 1986; 24: 38 - 44.

8 Zafonte RD, Demangone DA, Herbison GJ, Ditunno Jr, JF. Daily self care in quadriplegic subjects. NeuroRehabilitation 1991; 1: $17-24$.

9 Marino RJ et al. Assessing selfcare status in quadriplegia: comparison of the quadriplegia index of function (QIF) and the functional independence measure (FIM). Paraplegia 1993; 31: $225-233$.

10 American Spinal Injury Association. Standards for Neurological Classification of Spinal Cord Injury Patients. Chicago: American Spinal Injury Association, 1987.

11 American Spinal Injury Association. International Standards for Neurological and Functional Classification of Spinal Cord Injury. Revised 1992. Chicago: American Spinal Injury Association, 1992.

12 Cronbach LJ. Coefficient alpha and the internal structure of tests. Psychometrika 1951; 16: $297-334$.

13 Nunnally Jr JC. Psychometric Theory, 2nd ed. McGraw-Hill: New York. 1978. 
14 Streiner DL, Norman GR. Health measurement scales: a practical guide to their development and use. Oxford University Press: Oxford. 1989.

15 Marino RJ, Rider-Foster D, Maissel G, Ditunno JF. Superiority of motor level over single neurological level in categorizing tetraplegia. Paraplegia 1995; 33: 510-513.
16 Ditunno Jr JF, Cohen ME, Formal C, Whiteneck GG. Functional outcomes, In Stover SL, Whiteneck GG, DeLisa JA (eds) Spinal Cord Injury: Clinical Outcomes from the Model Systems. Gaithersburg, MD: Aspen, 1995, pp 170-184.

17 Kirshner B, Guyatt G. A methodoligical framework for assessing health indices. Journal of Chronic Disease 1985; 38: 27 - 36. 